\title{
Caracterização clínica e psicossocial da clientela de um ambulatório de esterilidade
}

\author{
Clinical and psychosocial characterization \\ of sterility clinic outpatients
}

\author{
Ricardo GORAYEB' \\ Andréa Cristina de Toledo BORSARI ${ }^{2}$ \\ Andreza Cristiana Ribeiro GOMES ${ }^{3}$ \\ Adriana Peterson Mariano Salata ROMÃO 4 \\ Rosana SHUHAMA ${ }^{2}$
}

\begin{abstract}
Resumo
Este estudo teve como objetivo descrever características demográficas, clínicas e psicossociais da clientela de um ambulatório de esterilidade e identificar motivação para atendimento psicológico. Tratou-se de estudo retrospectivo, com 50 casais, entrevistados no início do processo de investigação médica. Constatou-se que $80 \%$ dos casais estavam na primeira união conjugal, $64 \%$ tinham até oito anos de casados, $72 \%$ não tinham filhos e 78\% vinham tentando engravidar há no máximo seis anos. Desconheciam as causas da infertilidade 36\% dos casais, e 78\% nunca haviam se submetido a procedimentos de reprodução assistida. Demonstraram interesse em participar de grupo de apoio psicológico 94\% dos casais. Referências a medo de abortamento, malformação fetal e parto foram estatisticamente superiores aos medos relacionados à gravidez, procedimento de Reprodução Assistida e responsabilidades com o filho. Esses dados revelam variáveis importantes para a elaboração de intervenções fundamentadas no conhecimento da realidade dos pacientes.
\end{abstract}

Unitermos: Infertilidade. Psicologia da saúde. Reprodução assistida.

\begin{abstract}
The objective of this study was to describe the demographic, clinical and psychosocial characteristics of Sterility Clinic outpatients and to identify motivation for psychological care. It is a retrospective study, conducted using 50 couples, interviewed at the beginning of the medical inquiry process. Eighty percent of the couples were in their first marriage, 64\% had been married for up to 8 years, $72 \%$ had no children and $78 \%$ had been trying to conceive for up to 6 years. The causes of infertility were unknown in thirty-six percent

$\boldsymbol{\nabla \nabla \nabla \nabla}$

1 Universidade de São Paulo, Faculdade de Medicina de Ribeirão Preto, Departamento de Neurociências e Ciências do Comportamento, Serviço de Psicologia. Av. dos Bandeirantes, 3900, $3^{\circ}$ andar, 14049-900, Ribeirão Preto, SP, Brasil. Correspondência para/Correspondence to: R. GORAYEB. E-mail: $<$ rgorayeb@fmrp.usp.br>.

2 Universidade de São Paulo, Faculdade de Medicina de Ribeirão Preto, Hospital das Clínicas. Ribeirão Preto, SP, Brasil.

3 Universidade de São Paulo, Faculdade de Filosofia, Ciências e Letras de Ribeirão Preto. Ribeirão Preto, SP, Brasil.

4 Universidade de São Paulo, Faculdade de Medicina de Ribeirão Preto, Departamento de Ginecologia e Obstetrícia. Ribeirão Preto, SP, Brasil.

Agradecimento: a Gustavo Salata Romão pelo auxílio na análise estatística deste trabalho.
\end{abstract}


of the couples and 78\% had never being subjected to Assisted Reproduction procedures. Ninety-four percent of the couples showed an interest in participating in a psychological support group. The fear of abortion, fetal malformation and delivery was statistically more frequent than fears related to pregnancy, Assisted Reproduction procedures and the responsibilities of parenthood. These data reveal significant variables for the development of interventions based on the knowledge of patients' circumstances.

Uniterms: Infertility. Health psychology. Assisted reproduction.

O quadro clínico de infertilidade é definido como ausência de gravidez após um ano de atividade sexual regular sem práticas de anticoncepção. Na presença de fator de risco masculino ou feminino de infertilidade, esse tempo é menor (Ferriani \& Navarro, 2004). Os aspectos psicológicos da experiência de infertilidade têm sido discutidos de maneira relevante na literatura nacional e internacional. A infertilidade tem sido descrita como fonte de ansiedade para a maioria dos casais que a vivenciam, podendo desencadear sentimentos de inferioridade, raiva, culpa, depressão e frustração (Cousineau \& Domar, 2007; Domar, Zuttermeister, Seibel \& Benson, 1992; Farinati, Rigoni \& Müller, 2006; Moreira, Melo, Tomaz \& Azevedo, 2006; Serafini et al., 1998).

Considerando a investigação para diagnóstico médico, é possível observar que alterações da fertilidade são encontradas em ambos os parceiros com mais frequência do que se imagina. A dificuldade pode ser decorrente, na mulher, de disfunções ovulatórias, alterações da tuba uterina, abortamento espontâneo de repetição, endometriose pélvica, alterações genéticas, entre outras (Serafini et al., 1998). No homem, a infertilidade está relacionada à alteração dos parâmetros seminais (concentração, morfologia, motilidade e vitalidade), comprovada em pelo menos duas amostras de espermograma (Ferriani \& Navarro, 2004), podendo existir também obstrução dos ductos ejaculatórios ou ocorrência prévia de vasectomia.

Além desses fatores, observa-se em países desenvolvidos uma tendência à postergação do casamento e da concepção em função da busca por qualificação profissional da mulher e de maior estabilidade financeira do casal, situação que pode dificultar os planos de uma gravidez, uma vez que a idade feminina está relacionada à quantidade e qualidade de 288 óvulos.

\section{Infertilidade e procedimentos de reprodução assistida: aspectos psicológicos}

Para os casais que planejam filhos, o diagnóstico de infertilidade pode ocasionar um prejuízo nas relações sociais, profissional e conjugal, representando um obstáculo concreto à realização do projeto parental. Nessa direção, Cousineau e Domar (2007) assinalam o estresse e a falta de controle como intrínsecos a esse quadro, que também promove a estigmatização e a interrupção na trajetória para a vida adulta. Alesi (2005), por sua vez, acrescenta que a não concepção involuntária pode constituir uma crise importante na vida pessoal, trazendo tratamentos que mobilizam mudanças físicas e psicológicas para os casais.

Cwikel, Gidron e Sheiner (2004) afirmam que os problemas de fertilidade podem significar uma ofensa à autoestima, imagem corporal e autoavaliação para homens e mulheres que a vivenciam. O tratamento para infertilidade, por sua vez, pode ser longo e desgastante, e inclui diversos exames para a investigação de suas causas, entre eles, laboratoriais, físicos, ultrassonográficos, além de registro da temperatura corpórea basal, planejamento de relações sexuais, análise do muco cervical pós-coito e coleta de espermatozoide por masturbação, muitos desses considerados invasivos (McNaughton-Cassill et al., 2000).

Alguns estudos indicam que um período prolongado de tempo tentando engravidar, sem obtenção de sucesso, pode desencadear um desgaste para a qualidade do relacionamento conjugal e sexual (Chiba et al., 1997; Facchinetti, Matteo, Artini,Volpe \& Genazzani, 1997). Nesta direção, Chiba et al. (1997) consideram um período acima de quatro anos de tentativas como relevante para um potencial prejuízo.

Os procedimentos de reprodução assistida (RA), como a inseminação intrauterina, a fertilização in vitro (FIV) ou a injeção intracitoplasmática de espermatozoide, 
embora tenham provido muitos casais com uma nova oportunidade para a gravidez, também podem prolongar seu sofrimento e desapontamento, pois não oferecem garantia de sucesso. Assim, o casal percebe os mecanismos utilizados na busca de resolução de seu problema como insuficientes e a ansiedade e a frustração podem ser inevitáveis.

Tais procedimentos, associados à pressão social da família, amigos e conhecidos, podem gerar consequências psicológicas negativas, tais como aumento da ansiedade, depressão, conflitos matrimoniais, sentimentos de isolamento, raiva, fracasso pessoal, pessimismo, irritabilidade e deterioração do relacionamento sexual (Boivin, 1997; Collins, Freeman, Boxer \& Tureck, 1992; Domar et al., 1992; Downey et al., 1989; Facchinetti et al., 1997; Farinati et al., 2006; Newton, Hearn \& Yuzpe 1990).

Em um estudo para analisar características psicológicas e qualidade da relação conjugal de pacientes inférteis, realizado na China por Wang et al. (2007), observou-se que o stress relacionado à infertilidade e aos procedimentos de RA tinha um impacto negativo, avaliado por instrumentos psicológicos, sobre a saúde emocional e relacionamento conjugal das pacientes, quando comparado ao grupo controle. Para os autores, que defendem a atenção psicológica no cuidado ao quadro de infertilidade, a idade, a renda anual, a duração da infertilidade e o histórico de fracasso em procedimentos de fertilização têm correlação negativa com a saúde emocional e qualidade da relação conjugal.

Pottinger et al. (2006) estudaram as diferenças de gênero no enfrentamento da infertilidade e a associação entre enfrentamento e stress psicológico em casais que iriam se submeter a procedimentos de fertilização em um hospital universitário africano. O trabalho revelou que as mulheres pensavam, mais do que os homens, sobre o que haviam feito de errado para terem esse problema, e relatavam mais sobre sua dor para outras pessoas. Tais estratégias que estavam associadas a um nível de stress superior ao dos maridos.

Corrêa, Vizzotto e Cury (2007) procuraram avaliar, por meio de instrumento padronizado, a eficácia adaptativa de 57 casais brasileiros inseridos em um programa de RA, concluindo que, apesar de também demonstrarem dificuldades, os homens apresentaram melhor eficácia adaptativa do que as mulheres durante o programa de fertilização. Todavia, deve-se considerar que esse estudo foi realizado com mulheres inférteis e homens férteis.

Urrutia, Genghini e Makuch (2002) procuraram descrever o que as mulheres atendidas no Ambulatório de Reprodução Humana da Universidade Estadual de Campinas sentiam e pensavam a respeito de seu diagnóstico de esterilidade primária. A pesquisa, realizada com 30 pacientes, evidenciou que o diagnóstico foi sinônimo de surpresa e tristeza, além de fonte de cobrança interna, familiar e social. As autoras relataram que a maioria das pacientes manifestou ambivalência e desesperança em relação ao resultado do tratamento, $\mathrm{e}$ um terço referiu tranquilidade e esperança frente aos mesmos.

Boivin et al. (1998) procuraram examinar, prospectivamente, as reações de homens e mulheres durante cada estágio do ciclo de fertilização in vitro (FIV) a partir de um inventário diário de sintomas. Os resultados sugeriram um padrão similar de respostas entre os casais e indicaram que o fator que mais influenciou as reações durante a FIV foi a incerteza do resultado do procedimento. As mulheres pareceram ser mais sensíveis em relação a esta incerteza, mas ambos responderam com sentimentos de ambivalência, que envolvem estresse emocional e sentimentos de esperança.

Desta forma, é importante considerar a infertilidade como um quadro que requer atenção do psicólogo da saúde para o auxílio aos casais no enfrentamento dos problemas psicológicos a ela relacionados. Convém ressaltar que, mediante pesquisa bibliográfica realizada para a execução deste estudo (Medline e Lilacs, nos últimos 20 anos), não foram encontrados artigos cujo objetivo estivesse voltado à caracterização demográfica ou psicossocial de um ambulatório ou serviço de reprodução assistida, especialmente na América Latina, embora existisse a descrição demográfica desses pacientes em artigos que possuíssem outros objetivos.

\section{Aspectos inerentes ao serviço público}

Os procedimentos de RA requerem tecnologia e equipe especializadas, envolvendo um alto custo 
financeiro; desta forma, são desenvolvidos predominantemente em clínicas e centros particulares. $\mathrm{O}$ Hospital das Clínicas da Faculdade de Medicina de Ribeirão Preto da Universidade de São Paulo (HCFMRPUSP) foi um dos primeiros serviços públicos do Brasil a oferecer um tratamento completo de RA, em 1991, com a primeira gravidez ao final de 1992 (Ferriani et al., 1995). Para a viabilização do processo, o hospital responsabiliza-se pelos gastos envolvidos com a investigação clínica, materiais utilizados nos procedimentos, além de equipe especializada, e o casal arca com os custos referentes aos medicamentos.

A equipe multiprofissional do Ambulatório de Esterilidade (AEST) do HCFMRP-USP, vinculado ao Departamento de Ginecologia e Obstetrícia da Faculdade de Medicina de Ribeirão Preto da Universidade de São Paulo, é composta por médicos, biólogos, auxiliares de enfermagem, enfermeiros, biomédicos, assistentes sociais e psicólogos.

Os pacientes são atendidos no ambulatório após a Divisão Regional de Saúde agendar a consulta de triagem, mediante encaminhamento de Unidades Básicas de Saúde de municípios de diversos estados brasileiros. Essa consulta busca avaliar aspectos referentes ao quadro clínico de infertilidade, averiguando se o caso é eletivo para o AEST ou se existe alguma contraindicação médica para a realização do procedimento de RA. Além disso, neste momento, é realizada uma entrevista com a assistente social para avaliar se o casal apresenta condições mínimas de custeio das medicações. Após a consulta de triagem, inicia-se a fase de investigação da infertilidade, que consome aproximadamente oito meses, contando com a adesão de ambos os cônjuges para a realização dos diversos exames e retornos médicos. Concluída essa etapa, a equipe estará em condições de indicar a técnica de RA mais adequada à problemática do casal.

O Serviço de Psicologia do HCFMRP-USP atua no AEST com um psicólogo contratado e um residente do Programa de Aprimoramento Profissional em Psicologia Clínica e Hospitalar (PAPPCH) desse hospital. As intervenções psicológicas desenvolvidas procuram considerar os preceitos estabelecidos pela instituição, alicerçados na prática da assistência, pesquisa e ensino. Assim, a rotina assistencial, fundamentada em intervenções breves, individuais e grupais, é conjugada com a realização de pesquisas, que indagam sobre aspectos emocionais relacionados ao quadro de infertilidade, promovendo conhecimento, avaliação e reflexão sobre o trabalho do psicólogo nessa área. A prática de ensino é contemplada nas aulas teóricas, supervisões semanais e discussões de caso que o psicólogo contratado realiza com o residente do PAPPCH.

Considerando o quadro clínico de infertilidade conjugal como importante campo de atuação para a psicologia da saúde, este trabalho procurou descrever as características demográficas, clínicas e psicossociais da clientela de um ambulatório de esterilidade, pretendendo identificar motivações, medos e expectativas desses pacientes, além da demanda por atendimento psicoterápico, para que, assim, sejam proporcionadas intervenções psicológicas pertinentes às necessidades dos pacientes.

\section{Método}

Foi realizado um estudo retrospectivo baseado na análise quantitativa e qualitativa de entrevistas realizadas com casais do AEST após consulta médica no ambulatório, durante o ano de 2001. As entrevistas, de aproximadamente uma hora e trinta minutos de duração, eram parte da atividade assistencial psicológica, e eram realizadas pelo psicólogo contratado e um residente do PAPPCH seguindo um roteiro semiestruturado, elaborado pelo serviço de psicologia para investigar características demográficas, clínicas e psicossociais dos pacientes do AEST e averiguar a necessidade de atendimento psicoterápico individual ou grupal.

O roteiro semiestruturado contava com 35 questões abertas e 10 fechadas, que investigavam dados demográficos, nível de informação sobre a doença, nível de informação sobre os procedimentos de RA, dinâmica familiar e conjugal, além de motivação para ter filho e adotar. Também faziam parte do roteiro questões sobre as expectativas em relação aos procedimentos de RA, relacionadas ao bebê, à equipe médica e à gravidez. Foram investigados os medos referentes aos procedimentos de RA, aborto, gravidez, parto e bebê, além da motivação para participar de grupo de apoio psicológico e da existência de acompanhamento psicológico e psiquiátrico prévios. 
Durante o ano de 2001 foram entrevistados 113 casais do Ambulatório de Esterilidade. Com a finalidade de realizar um estudo de caracterização deste ambulatório, foram selecionados 87 casais; foram descartadas 26 entrevistas em que haviam questões não respondidas ou incompletas (dados perdidos). Assim, foi encaminhado um projeto de pesquisa ao Comitê de Ética do HCFMRP-USP solicitando autorização para utilização dos dados da entrevista mediante assinatura de Termo de Consentimento Livre e Esclarecido (TCLE) pelos pacientes. Após aprovação do Comitê de Ética, os 87 casais foram contatados por telefone para explicação do referido projeto; na sequência, foi enviada uma correspondência contendo o TCLE e uma carta-resposta selada, para que os casais devolvessem o TCLE assinado. O estudo foi realizado com 50 casais, ou seja, com aqueles que responderam à carta contendo o TCLE.

Os dados coletados com o roteiro de entrevista foram categorizados e analisados quantitativa e qualitativamente. Para questões fechadas, foi realizada análise quantitativa por meio do programa estatístico Prisma 3.0, utilizando-se o teste não paramétrico de Fischer. Para as questões abertas, inicialmente foi empregada análise qualitativa de dados, utilizando a análise de conteúdo temática proposta por Bardin (1977) e Minayo (1994), seguida de análise quantitativa (com utilização do teste Qui-Quadrado), em questões nas quais havia o interesse de verificar diferenças estatisticamente significativas entre homens e mulheres $(p \leq 0,5)$.

\section{Resultados}

As mulheres tinham idade média de 30,8 (desvio-padrão - $\mathrm{DP}=4,98)$ e os homens de 33,5 (DP=6,81) anos. A maioria dos casais (82\% das mulheres, $n=41 ; 80 \%$ dos homens, $n=40$ ) declarou estar vivendo sua primeira união conjugal; $78 \%$ deles ( $n=39$ ) eram casados e 22\% ( $n=11)$ viviam em união estável. Em relação à procedência, 30\% dos casais ( $n=15)$ residiam em Ribeirão Preto, 54\% ( $n=27$ ) moravam até $140 \mathrm{~km}$ de distância e $16 \%(n=8)$ provinham de localidades mais distantes. No que se refere à escolaridade, $42 \%$ das mulheres $(n=21)$ e dos homens apresentavam de 9 a 11 anos de estudo, 0 que corresponde ao ensino médio completo ou incompleto. Quanto à situação econômica, foi observado que para $8 \%$ dos casais $(n=4)$ a renda mensal relatada foi de
1,0 a 3,0 salários-mínimos, 66\% ( $n=33$ ) referiram de 3,1 a $9,0$ salários-mínimos e $26 \%$ ( $n=13)$ afirmaram receber acima de 9,1 salários-mínimos; para 44\% das mulheres ( $n=22$ ) e 64\% dos homens ( $n=32)$, o trabalho relacionava-se ao nível primário de produção.

Entre os casais analisados, 94\% ( $n=47)$ informaram a familiares e amigos mais próximos sobre o quadro clínico de infertilidade conjugal. A respeito da qualidade da influência social que sofriam, 44\% ( $n=22)$ relataram incentivos em relação à busca por filhos, 24\% $(n=12)$ citaram o apoio social concomitante à cobrança, 22\% $(n=11)$ disseram sentir-se cobrados socialmente pela ausência de filhos e 10\% afirmaram que a opinião alheia não tinha relevância em suas vidas. No que tange à religiosidade, $72 \%$ dos casais $(n=36)$ declararam-se católicos, 18\% ( $n=9)$ evangélicos, 8\% ( $n=4)$ espíritas e $2 \%(n=1)$ referiram não ter religião definida.

O tempo de casamento relatado pelos casais foi de até oito anos para $64 \%$ dos pares $(n=32)$, e acima de oito anos para o restante. Quanto à experiência de maternidade/paternidade, $82 \%$ das mulheres $(n=41)$ e $72 \%$ dos homens ( $n=36)$ afirmaram não ter filhos; os demais referiram ter filhos desta ou de relações anteriores. No que diz respeito ao período de tempo em que estavam tentando engravidar, $48 \%$ dos casais ( $n=24)$ relataram um período de, no máximo, quatro anos de tentativas; outros 30\% $(n=15)$ referiram estar de 4 a 6 anos tentando conceber.

Quando questionados a respeito da qualidade da relação conjugal, 52\% ( $n=26$ ) dos casais consideraram seu relacionamento afetivo ótimo, 44\% ( $n=22)$ declararam-no bom e $4 \%(n=2)$ julgaram-no regular. A área sexual foi avaliada como ótima por $36 \%$ dos casais $(n=18)$, considerada boa por $60 \%$ deles $(n=30)$ e regular para apenas 4\% $(n=2)$ dos entrevistados.

Em relação ao quadro clínico de infertilidade conjugal, $36 \%$ das mulheres ( $n=18$ ) e $40 \%$ dos homens $(n=20)$ relataram desconhecer as causas. Entre as entrevistadas que tinham conhecimento de seu quadro clínico, 16\% ( $n=8$ ) referiram obstrução tubária, 12\% (n=6) mencionaram síndrome do ovário policístico, 10\% ( $n=5)$ relataram endometriose e $6 \%(n=3)$ já haviam realizado a laqueadura tubária. Entre os parceiros, 30\% ( $n=15)$ disseram que existiam alterações no exame de espermograma, quer fossem vinculadas à motilidade, concentração, vitalidade ou qualidade, $2 \%(n=1)$ haviam 
apresentado câncer de próstata e 2\% ( $n=1$ ) tinham realizado vasectomia. Além desses fatores, $12 \%$ das mulheres $(n=6)$ e $26 \%$ dos homens $(n=13)$ afirmaram que os exames prévios não detectaram nenhuma patologia relevante que justificasse a infertilidade.

Em relação aos procedimentos de RA, 78\% dos casais ( $n=39)$ afirmaram nunca tê-los realizado, 16\% $(n=8)$ submeteram-se ao processo uma vez, enquanto $6 \%(n=3)$ passaram por essa experiência mais de uma vez. Quanto ao conhecimento a respeito dos procedimentos, $6 \%$ das mulheres $(n=3)$ e $14 \%$ dos homens $(n=7)$ manifestaram um conhecimento satisfatório dos procedimentos, $32 \%$ das mulheres ( $n=16$ ) e $26 \%$ dos homens ( $n=13)$ revelaram um conhecimento parcial dos procedimentos, $22 \%$ das mulheres $(n=11)$ e $12 \%$ dos homens $(n=6)$ estavam pouco informados do assunto, além do relato de total falta de informação na área para $40 \%$ das mulheres ( $n=20)$ e $48 \%$ dos homens $(n=24)$.

Quando indagados sobre a motivação para ter um filho, 36\% das mulheres $(n=18)$ e $18 \%$ dos homens $(n=9)$ afirmaram que um filho fazia falta em suas vidas, com relatos como: "Não falta mais nada para nós, falta só uma criança" ou "Casalégostoso, mas chega uma hora que falta alguma coisa". Há significativamente mais mulheres do que homens apresentando esta resposta. Já para $18 \%$ das mulheres $(n=9)$ e $12 \%$ dos homens $(n=6)$, a ideia de ter um filho foi algo que ocorreu naturalmente, após algum tempo de casamento, como assim descrito: "Eu acho que isso é uma coisa natural depois que o casal decide que vai ficar junto". Além disso, existiram razões específicas para cada gênero, por exemplo, para $14 \%$ dos homens $(n=7)$ a questão de continuidade do sobrenome foi citada e para $4 \%$ das mulheres $(n=2)$, a demanda foi marcada pela sua idade.

A respeito das mudanças ocorridas com a chegada do filho, $28 \%$ das mulheres $(n=14)$ e $20 \%$ dos homens $(n=10)$ acreditavam que ficariam mais alegres e/ou felizes, apresentando verbalizações como: "Eu acho que ter um filho vai ser só alegria, é a satisfação maior" ou "Vai ter mais alegria na vida da gente, nós vamos sentir a vida completa". Por outro lado, $18 \%$ das mulheres $(n=9)$ e $26 \%$ dos homens ( $n=13)$ manifestaram preocupação em relação às responsabilidades que um filho traz, afirmando que "Muitas coisas vão mudar, vai ser uma preocupação maior, tudo vai girar em torno da criança, para o bem dela" ou "Eu acho que muda, vai mudar as responsabilidades, a gente vai ter que pensar mais nas coisas".

Além disso, para 24\% das mulheres $(n=12)$ e $8 \%$ dos homens $(n=4)$, a chegada do filho acarretaria alterações importantes na rotina do casal, modificando horários e o ritmo de vida presente, o que pode ser percebido nos seguintes relatos: "Com o filho, altera toda a rotina de uma casa. Em termos de horário, de alimentação, passeio, vai dar um giro de 360 graus" ou "Tudo, porque atéagora foi uma vida para dois. A casa agora fica arrumadinha, comida na hora certa, mas depois com o bebênão vai ter hora mais para nada..." Embora apresentando frequência diversa nos relatos acima, não houve diferença estatisticamente significativa nas respostas apresentadas entre mulheres e homens nesta questão. NaTabela 1 é possível visualizar as diferenças de opinião entre homens e mulheres no que se refere à motivação e expectativa a respeito do filho.

Quando questionados a respeito da adoção, 32\% das mulheres ( $n=16)$ e $26 \%$ dos homens $(n=13)$ afirma-

Tabela 1. Frequência e porcentagem do relato de casais do Ambulatório de Esterilidade do Hospital das Clínicas da Faculdade de Medicina de Ribeirão Preto, da Universidade de São Paulo, sobre motivação e expectativa em relação ao filho. (2001).

\begin{tabular}{|c|c|c|c|c|c|}
\hline \multirow{2}{*}{\multicolumn{2}{|c|}{ Aspectos psicossoais }} & \multicolumn{2}{|c|}{ Mulher } & \multicolumn{2}{|c|}{ Homem } \\
\hline & & Frequência & $\%$ & Frequência & $\%$ \\
\hline \multirow{4}{*}{ Motivação para ter um filho } & Filho é algo que faz falta & $18^{*}$ & 36 & $9^{*}$ & 18 \\
\hline & Filho é algo esperado após o casamento & 9 & 18 & 6 & 12 \\
\hline & Continuidade do sobrenome & 0 & 0 & 7 & 14 \\
\hline & Idade & 2 & 4 & 0 & 0 \\
\hline \multirow{3}{*}{ Expectativa } & Felicidade, Alegria & 14 & 28 & 10 & 20 \\
\hline & Preocupações & 9 & 18 & 13 & 26 \\
\hline & Alterações na rotina & 12 & 24 & 4 & 8 \\
\hline
\end{tabular}

*Diferença estatisticamente significativa (teste Qui-Quadrado). 
ram que adotariam uma criança apenas se não conseguissem ter o filho biológico, $26 \%$ das mulheres $(n=13)$ e $32 \%$ dos homens $(n=16)$ disseram que não pretendiam adotar e, para 20\% das mulheres $(n=10)$ e 16\% dos homens ( $n=8)$, a adoção era percebida como algo positivo e possível de acontecer. Além disso, 22\% das mulheres ( $n=11)$ e $26 \%$ dos homens $(n=13)$ relataram não ter uma opinião formada sobre o tema ou não ter pensado no assunto.

Ao analisar a Tabela 2, constata-se que os medos relatados com maior frequência pelos casais referiam-se ao parto, à possibilidade de aborto e riscos à saúde do bebê, como malformação fetal e doenças congênitas. Os relatos desses medos foram estatisticamente superiores aos demais medos referidos. É fundamental salientar que não existiram diferenças estatisticamente significativas entre as frequências dos medos relatados por homens e mulheres. A frequência do medo referente ao aborto mostrou-se estatisticamente superior àquelas relacionadas aos medos da gravidez, do procedimento de RA e de responsabilidades em relação à maternidade/paternidade $(p<0,01)$. O medo em relação à saúde do bebê apresentou-se estatisticamente superior aos medos de gravidez, do procedimento de RA e de responsabilidades $(p<0,01)$. Além disso, o medo do parto mostrou-se estatisticamente superior aos medos vinculados à gravidez, procedimento de RA e de responsabilidades $(p<0,01)$. Em relação à experiência de aborto, foi analisada a frequência do relato de medo dessa ocorrência, observando-se não haver diferença estatística entre os dez casais que a vivenciaram, quando

Tabela 2. Frequência e porcentagem dos medos relatados por membros dos casais em investigação no Ambulatório de Esterilidade do Hospital das Clínicas da Faculdade de Medicina de Ribeirão Preto, da Universidade de São Paulo. (2001).

\begin{tabular}{|c|c|c|c|c|}
\hline \multirow{2}{*}{ Medos } & \multicolumn{2}{|c|}{ Mulher } & \multicolumn{2}{|c|}{ Homem } \\
\hline & Frequência & $\%$ & Frequência & $\%$ \\
\hline Riscos do parto* & 20 & 40 & 15 & 30 \\
\hline Aborto* & 23 & 46 & 18 & 36 \\
\hline Referente ao bebê* & 23 & 46 & 17 & 34 \\
\hline Gravidez & 5 & 10 & 1 & 2 \\
\hline Riscos do procedimento & 1 & 2 & 1 & 2 \\
\hline Responsabilidades & 1 & 2 & 2 & 4 \\
\hline
\end{tabular}

* Diferenças estatisticamente superiores quando comparadas aos demais medos. comparados com os casais que nunca passaram por esta realidade.

Quanto à motivação para participar de um grupo informativo e de apoio psicológico pré-fertilização, 94\% dos casais ( $n=47$ ) demonstraram interesse em comparecer, tendo os $6 \%$ restantes $(n=3)$ relatado impossibilidade de frequentá-lo devido ao trabalho ou à distância de sua cidade. Em relação à existência de atendimento psicológico prévio, foi observado que 84\% das mulheres ( $n=42)$ mencionaram não ter se submetido a qualquer tratamento psicológico, relato que, no caso dos homens, sobe para 94\% $(n=47)$. A respeito de atendimento psiquiátrico, foi verificado que esse nunca ocorreu para 92\% das mulheres $(n=46)$ e $94 \%$ dos homens $(n=47)$.

\section{Discussão}

Este trabalho procurou descrever as características demográficas, clínicas e psicossociais de pacientes de um ambulatório de esterilidade, incluindo motivações, medos e expectativas desses indivíduos, além de investigar o interesse por atendimento psicoterápico neste cenário. Os sujeitos deste estudo trabaIhavam, em grande parte, no nível primário de produção, apresentando níveis de renda que oscilavam de três a nove salários-mínimos, apesar de possuírem um nível de instrução superior à média brasileira. Contudo, considerando o custo oneroso dos procedimentos de RA no setor privado e a baixa taxa de sucesso desses, é compreensível a procura desse tipo de tratamento no serviço público pela grande maioria destes pacientes.

Chiba et al. (1997) consideram um período acima de quatro anos de tentativas relevante para um potencial prejuízo da qualidade do relacionamento conjugal e sexual. No estudo aqui descrito, constatou-se que a maioria dos casais avaliou seu relacionamento conjugal como satisfatório, classificando-o como ótimo e bom, sinalizando, assim, a ausência do relato de dificuldades conjugais relevantes, apesar de estarem, na maioria, tentando engravidar há mais de quatro anos. É importante considerar, contudo, que os cônjuges foram entrevistados em conjunto, o que pode ter contribuído para uma avaliação idealizada do relacionamento, com 
apreciações positivas devido à presença do parceiro. Soma-se a isso o fato de a entrevista ter sido realizada por um membro da equipe, o psicólogo, que embora explicite seu papel de apoio e acolhimento aos casais, também pode ser percebido como alguém que poderia excluí-los do atendimento médico em função de uma avaliação negativa do relacionamento conjugal. No que se refere à qualidade do relacionamento sexual, foi observado que as avaliações não foram tão positivas quanto às apresentadas para a relação afetiva. Uma possível explicação para isso seria o quadro clínico de infertilidade, que impõe datas e horários programados para o coito, sucessivas tentativas de gravidez e repetidas vivências de fracasso, condicionando ainda mais a relação que se estabelece entre sexo e fecundação, em detrimento do prazer.

Em relação ao apoio social, foi observado que a grande maioria, $94 \%$ dos casais $(n=47)$, referiu ter comunicado a familiares ou amigos sobre a infertilidade conjugal, o que pode indicar que socialmente existe algum grau de compartilhamento do problema. Ao avaliar a qualidade da influência que sofrem, os casais expõem uma postura de apoio e incentivo ao lado da típica cobrança por filhos para aqueles que não os têm, presentes nos relatos de $46 \%$ ( $n=23)$ da amostra, o que ressalta a ideia da infertilidade como fonte de estresse nas relações sociais.

\section{Considerações Finais}

Os dados também apontam o desconhecimento das causas de infertilidade conjugal para uma parcela considerável dos casais, bem como a falta de informação em relação aos procedimentos de RA para a maioria deles, talvez justificados pelo fato de $78 \%$ dos entrevistados ( $n=39$ ) nunca haverem se submetido a qualquer técnica de RA e estarem no início do processo e contato com a equipe. Esses dados sinalizam a necessidade de intervenções específicas da equipe multidisciplinar que favoreçam o fornecimento de informações e esclarecimentos médicos aos casais.

No que se refere à expectativa de ter um filho, observou-se que as respostas apresentadas pelas pacientes estiveram relacionadas à crença de que ficariam mais felizes com a chegada deste e à percepção das alterações importantes na rotina do casal. Os homens, por sua vez, manifestaram preocupação em relação às responsabilidades advindas da paternidade; também acreditavam que ficariam mais felizes, mas poucos relataram sobre a alteração da rotina para o casal. Assim, nota-se que as mulheres percebiam mais claramente as alterações na rotina conjugal e os homens ressaltaram as responsabilidades, sobretudo financeiras, derivadas da vinda do filho.

As motivações femininas e masculinas estiveram vinculadas à falta do filho em suas vidas e à ideia do planejamento desse após o casamento. Além disso, os homens citaram a questão da continuidade do sobrenome, não referida pelas mulheres, mais atentas à idade. Foi observado que as mulheres apresentaram significativamente maior frequência do relato de que o filho fazia falta em sua vida, o que pode estar relacionado a fatores culturais, que associam a ideia de maternidade às de completude e felicidade, como pode ser observado no estudo de Trindade e Enumo (2002).

Em relação à adoção, notou-se que a maioria de homens e mulheres afirmou não desejar adotar, ou que adotariam apenas se não conseguissem êxito nos procedimentos de RA. Esta questão merece uma investigação mais detalhada, no sentido de averiguar se, com a disponibilidade e diversidade de técnicas de reprodução assistida, a procura por adoção declinou em casais que não engravidam naturalmente.

A maioria dos estudos enfatiza a importância da atenção psicológica ao casal infértil, uma vez que ansiedades e medos são observados em ambos os cônjuges. Neste trabalho, foi verificado que os medos referentes ao parto, aborto e riscos relacionados à saúde do bebê foram acentuados em homens e mulheres, não havendo diferenças estatísticas entre os cônjuges, o que ratifica a ideia de que ambos necessitam igualmente de apoio psicológico. Nesta direção, tendo em vista os medos salientados, seria oportuna a avaliação do nível de ansiedade e depressão desta clientela, mais precisamente por meio de instrumentos que possam avaliar a existência destas dificuldades vinculadas à infertilidade. Porém, até o momento, não há instrumento com essa finalidade criado ou adaptado para a língua portuguesa.

A respeito do apoio psicológico, é possível perceber que $94 \%$ dos casais $(n=47)$ avaliaram positivamente o auxílio nessa área, embora a grande maioria 
de mulheres e homens tenha mencionado que nunca se submeteu a qualquer tratamento psicológico ou psiquiátrico. Por outro lado, é observada na prática ambulatorial uma baixa demanda por psicoterapia, o que torna necessários novos estudos que possibilitem a compreensão deste fenômeno e a extensão deste serviço aos pacientes necessitados.

Os dados coletados neste estudo podem contribuir para o conhecimento do contexto sociodemográfico e psicossocial no qual casais inférteis em busca de reprodução assistida estão inseridos, permitindo a elaboração de propostas de atendimento psicológico adequadas ao perfil dessa clientela. Convém enfatizar que os dados encontrados podem ser aplicados apenas à população descrita, e não generalizados amplamente.

Nesta direção, sugere-se que grupos de apoio psicológico aos casais com diagnóstico de infertilidade possam ser eficientes para a redução dos medos e ansiedades relatados. Variáveis como nível de informação sobre o quadro clínico e procedimentos de RA, apoio e cobrança social, motivação para ter filho, relacionamento conjugal e sexual e expectativas e medos referentes aos procedimentos de RA devem ser consideradas nas intervenções psicológicas. É fundamental que atendimentos psicológicos em grupo ou individuais possam ser oferecidos a casais ou pacientes que possuam demanda para tal, bem como o desenvolvimento de intervenções que conciliem o atendimento psicológico focal à informação médica, visando esclarecimentos e informações a respeito do quadro de infertilidade conjugal e procedimentos de RA. Além disso, grupos de apoio específico para casais onde há histórico de aborto de repetição seriam desejáveis, considerando-se as ansiedades referidas pelos casais que apresentam esse quadro clínico.

\section{Referências}

Alesi, R. (2005). Infertility and its treatment: an emotional roller coaster. Australian Family Physician, 34 (3), 135-138.

Bardin, L. (1977). Análise de conteúdo. Lisboa: Persona.

Boivin, J. (1997). Is there too much emphasis on psychosocial counseling for infertile patients? Journal of Assisted Reproduction and Genetics, 14 (4), 184-186.

Boivin, J., Andersson, L., Skoog-Svanberg, A., Hjelmstedt, A. Collins, A., \& Bergh, T. (1998). Psychological reactions during in vitro fertilization: similar response pattern in husbands and wives. Human Reproduction, 13 (11) 3262-3267.

Chiba, H., Mori, E., Morioka, Y., Kashiwakura, M., Nadaoka, T., Saito, H., \& Hiroi, M. (1997). Stress of female infertility: relations to lenght of treatment. Gynecology Obstetric Investigation, 43 (3), 171-177.

Collins, A., Freeman, E. W., Boxer, A. S., \& Tureck, R. (1992). Perceptions of infertility and treatment stress in females as compared with males entering in vitro fertilization treatment. Fertility and Sterility, 57 (2), 350-356.

Corrêa, K. R. F. D'C., Vizzotto, M. M., \& Cury, A. F. (2007). Avaliação da eficácia adaptativa de mulheres e homens inseridos num programa de fertilização in vitro. Psicologia em Estudo, 12 (2), 363-370.

Cousineau, T. M., \&Domar, A. D. (2007). Psychological impact of infertility. Best Practice \& Research Clinical Obstetrics and Gynaecology, 21 (2), 293-308.

Cwikel, J., Gidron, Y., \& Sheiner, E. (2004). Psychological interactions with infertility among women. European Journal of Obstetrics \& Gynecology and Reproductive Biology, 117 (2004), 126-131.

Domar, A. D., Zuttermeister, M. A., Seibel, M., \& Benson, H. (1992). The prevalence and predictability of depression in infertile women. Fertility and Sterility, 58 (6), 1158-1163.

Downey, J., Yingling, S., McKinney, M., Husami, N., Jewelewicz, R., \& Maidman, J. (1989). Mood disorders, psychiatric symptoms, and distress in women presenting for infertility evaluation. Fertility and Sterility, 52 (3), 425-432.

Facchinetti, F., Matteo, M. L., Artini, G. P., Volpe, A., \& Genazzani, A. R. (1997). An increased vulnerability to stress is associated with a poor outcome of in vitro fertilization-embryo transfer treatment. Fertility and Sterility, 67 (2), 309-314.

Farinati, D. M., Rigoni, M. S., \& Muller, M. C. (2006). Infertilidade: um novo campo da psicologia da saúde. Estudos de Psicologia (Campinas), 23 (4), 433-439.

Ferriani, R. A., \& Navarro, P. A. A. S. (2004). Protocolos de condutas em infertilidade conjugal. São Paulo: Conexão Brasil.

Ferriani, R. A., Moura, M. D., Sala, M. M., Yamazaki, R., Reis, R. M., \& Sá, M. F. S. (1995). Fertilidade assistida no Hospital das Clínicas de Ribeirão Preto (HCRP). Reprodução e Climatério, 10 (4), 151-152.

McNaughton-Cassill, M. E., Bostwick, M., Vanscoy, S. E., Arthur, N. J., Hickman, T. N., Robinson, R. D., \& Neal, G.S. (2000). Development of brief stress management support groups for couples undergoing in vitro fertilization treatment. Fertility and Sterility, 74 (1), 87-93.

Minayo, M. C. S. (1994). O desafio do conhecimento: pesquisa qualitativa em saúde. São Paulo: Hucitec.

Moreira, S. N. T., Melo, C. O. M., Tomaz, G., \& Azevedo, G. D. (2006). Estresse e ansiedade em mulheres inférteis. Revista Brasileira Ginecologia Obstetrícia, 28 (6), 358-364.

Newton, C. R., Hearn, M. T., \& Yuzpe, A. A. (1990). Psychological assessment and follow-up after in vitro fertilization: 
assessing the impact of failure. Fertility and Sterility, 54 (5), 879-886.

Pottinger, A. M, McKenzie, C., Fredericks, J., DaCosta, V., Wynter, S., Everett, D., \& Walters, Y. (2006). Gender differences in coping with infertility among couples undergoing counselling for in vitro fertilization treatment. West Indian Medical Journal, 55 (4), 237-242.

Serafini, P., White, J., Petracco, A., Motta, E., Yadid, I. M., \& Coslovsky, M. (1998). O bê a bá da infertilidade. São Paulo: Organon.

Trindade, Z. A., \& Enumo, S. R. F. (2002). Triste e incompleta: uma visão feminina da mulher infértil. Psicologia USP, 13 (2), 151-182.
Urrutia, D. N., Genghini, M. H. R. R., \& Makuch, M. Y. (2002). Vivências de mulheres com diagnóstico de infertilidade primária: significado para o atendimento ambulatorial. Reprodução \& Climatério, 17 (2), 118-121.

Wang, K., L., J., Zhang, J. X., Zhang, L., Yu, J., \& Jiang, P. (2007). Psychological characteristics and marital quality of infertile women registered for in vitro fertilization-intracytoplasmic sperm injection in China. Fertility and Sterility, 87 (4), 792-798.

Recebido em: 6/8/2007

Versão final reapresentada em: 30/7/2008

Aprovado em: 29/9/2008 